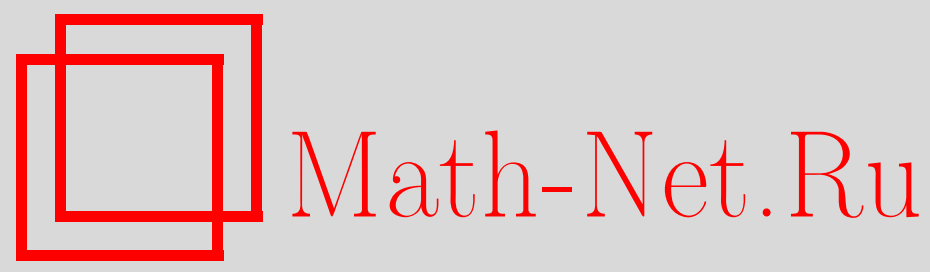

О. В. Матвеев, Об одном методе интерполирования функций на хаотических сетках, Матем. заметки, 1997, том 62, выпуск 3, 404-417

DOI: https://doi.org/10.4213/mzm1622

Использование Общероссийского математического портала Math-Net.Ru подразумевает, что вы прочитали и согласны с пользовательским соглашением http://www . mathnet.ru/rus/agreement

Параметры загрузки:

IP: 54.147 .182 .235

26 апреля 2023 г., $11: 16: 55$

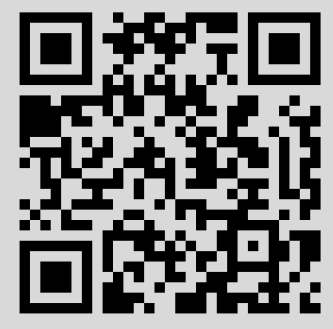




\section{ОБ ОДНОМ МЕТОДЕ ИНТЕРПОЛИРОВАНИЯ ФУНКЦИЙ НА ХАОТИЧЕСКИХ СЕТКАХ \\ О.В. Матвеев}

Пусть $m, n \in \mathbb{N}, m \equiv n(\bmod 2), K(x)=|x|^{m}$ при нечетном $m, K(x)=|x|^{m} \ln |x|$ при четном $m\left(x \in \mathbb{R}^{n}\right), \mathscr{P}$ - множество вещественных полиномов от $n$ переменных степени $\leqslant m / 2$ по совокупности переменных и $x_{1}, \ldots, x_{N} \in \mathbb{R}^{n}$. Строится функция вида

$$
\sum_{j=1}^{N} \lambda_{j} K\left(x-x_{j}\right)+P(x), \quad \text { где } \lambda_{j} \in \mathbb{R}, \quad P \in \mathscr{P}, \quad \sum_{j=1}^{N} \lambda_{j} Q\left(x_{j}\right)=0 \quad \forall Q \in \mathscr{P},
$$

совпадающая с заданной функцией $f(x)$ в точках $x_{1}, \ldots, x_{N}$. Для этого метода интерполирования получены оценки погрешности приближения функций $f \in W_{p}^{k}(\Omega)$ и их производных порядка $l$ в нормах $L_{q}\left(\Omega_{\varepsilon}\right)$, где $\Omega$ - ограниченная область в $\mathbb{R}^{n}, \varepsilon>0$, $\Omega_{\varepsilon}=\{x \in \Omega: \operatorname{dist}(x, \partial \Omega)>\varepsilon\}$.

Библиографоия: 14 названий.

Хорошо изученным методом приближения функций одной переменной является интерполирование кусочно-полиномиальными сплайнами $s(x)$ нечетной степени $m$ дефекта 1 (т.е. класса $C^{m-1}$ ) на сетке узлов $x_{1}<x_{2}<\cdots<x_{N}$, удовлетворяющими краевым условиям $s^{(l)}\left(x_{1}\right)=s^{(l)}\left(x_{N}\right)=0$ при $(m+1) / 2 \leqslant l \leqslant m-1$ (см. [1], [2]). Такой сплайн может быть представлен в виде

$$
\sum_{j=1}^{N} \lambda_{j}\left|x-x_{j}\right|^{m}+P(x)
$$

где $\lambda_{j} \in \mathbb{R}, P$ - полином степени $\leqslant(m-1) / 2$,

$$
\sum_{j=1}^{N} \lambda_{j} Q\left(x_{j}\right)=0
$$

для любого полинома $Q$ степени $\leqslant(m-1) / 2$. Мы рассмотрим следующее обобщение этого метода на случай $n$ переменных. Пусть $m, n \in \mathbb{N}, m \equiv n(\bmod 2), K(x)=|x|^{m}$

Работа выполнена при финансовой поддержке Российского фонда фундаментальных исследований, гранты № 95-01-00287a, № 93-011-196. 
при нечетном $m$ и $K(x)=|x|^{m} \ln |x|$ при четном $m\left(x \in \mathbb{R}^{n}\right)$, при $t \geqslant 0 \mathscr{P}_{t}$ - множество полиномов (вещественных) от $n$ переменных степени $\leqslant t$ по совокупности переменных, при $t<0 \mathscr{P}_{t}=\{o\}$,где $o$-нулевой полином, $\Delta=\left\{x_{1}, \ldots, x_{N}\right\}$-произвольноенепустое конечное подмножество $\mathbb{R}^{n}$. Строится функция вида

$$
\begin{gathered}
s(x)=\sum_{j=1}^{N} \lambda_{j} K\left(x-x_{j}\right)+P(x), \\
\lambda_{j} \in \mathbb{R}, \quad P \in \mathscr{P}_{m / 2}, \quad \sum_{j=1}^{N} \lambda_{j} Q\left(x_{j}\right)=0 \quad \forall Q \in \mathscr{P}_{m / 2},
\end{gathered}
$$

совпадающая с заданной функцией $f(x)$ в точках $x_{1}, \ldots, x_{N}$. Такой метод интерполирования рассматривался ранее Ж. Дюшоном[3]. В данной статье исследуются аппроксимативные свойства функций (1). Пусть $\Omega$ - ограниченная область в $\mathbb{R}^{n}$, удовлетворяющая сильному условию конуса $[4$, с. 118] (или, что эквивалентно, ограниченная область с минимально гладкой границей [5, с. 224]). Заметим, что функция (1) имеет наиболее простой вид при $m=1$

$$
s(x)=\sum_{j=1}^{N} \lambda_{j}\left|x-x_{j}\right|+c, \quad \lambda_{j}, c \in \mathbb{R}, \quad \sum_{j=1}^{N} \lambda_{j}=0
$$

("multi-conic functions" в [3]). Из наших результатов следует, в частности, что при нечетном $n$ существует единственная функция $s_{1}(f, \Delta)$ вида $(2)$, интерполирующая $f$ на $\Delta$, и если $f$ непрерьвна и имеет ограниченные обобщенные производные порядка $n+1$ на $\Omega, \Delta_{j}$ - непустое конечное подмножество $\Omega(j \in \mathbb{N})$,

$$
h_{j}=\sup _{x \in \Omega} \inf _{y \in \Delta_{j}}|x-y| \rightarrow 0 \quad \text { при } j \rightarrow \infty
$$

то для любой точки $x \in \Omega f(x)-s_{1}\left(f, \Delta_{j}\right)(x)=O\left(h_{j}^{n+1}\right)$ при $j \rightarrow \infty$. Таким образом, порядок поточечной сходимости функций $(2)$ к достаточно гладкой интерполируемой функции $f$ возрастает вместе с размерностью.

Мы будем использовать следующие обозначения: $\mathbb{Z}_{+}=\mathbb{N} \cup\{0\} ; \mu=(m+n) / 2$, $m^{\prime}=[m / 2]+1$; если $\alpha=\left(\alpha_{1}, \ldots, \alpha_{n}\right)$ - мультииндекс с цельми неотрищательными компонентами и $x=\left(e_{1}, \ldots, e_{n}\right) \in \mathbb{R}^{n}$, то $|\alpha|=\alpha_{1}+\cdots+\alpha_{n}, \alpha$ ! $=\alpha_{1} ! \cdots \alpha_{n}$ !, $x^{\alpha}=e_{1}^{\alpha_{1}} \cdots e_{n}^{\alpha_{n}}$ (считаем $\left.\mathbf{0}^{0}=1\right) ; B(a, r)=\left\{x \in \mathbb{R}^{n}:|x-a|<r\right\}, B_{\zeta}(a, r)=B(a, r) \cap \zeta \Omega$, $\operatorname{dist}\left(F, F^{\prime}\right)=\inf \left\{|x-y|: x \in F, y \in F^{\prime}\right\}, \operatorname{dist}(a, F)=\operatorname{dist}(\{a\}, F)$,

$$
\bar{h}(F)=\sup _{x \in \Omega} \operatorname{dist}(x, F), \quad \underline{h}(F)=\inf \{|x-y|: x, y \in F, x \neq y\}
$$

$\left(\underline{h}(F)=+\infty\right.$, если $F$ состоит из одной точки), $\Omega_{r}=\{x \in \Omega: \operatorname{dist}(x, \partial \Omega)>r\}$, где $a \in \mathbb{R}^{n}, r, \zeta>0, F, F^{\prime} \subset \mathbb{R}^{n}, F, F^{\prime} \neq \varnothing ; \mathscr{A}_{t}$ - множество таких множеств $F \subset \mathbb{R}^{n}$, что $\left(P \in \mathscr{P}_{t}, P(x)=0 \forall x \in F\right) \Longrightarrow(P(x) \equiv 0)$, где $t \in \mathbb{R} ; \mathscr{L}$ - оператор Лапласа, $\mathscr{F}$ - преобразование Фурье; $\mathscr{D}(G)$ - множество бесконечно дифференцируемых функций $g: \mathbb{R}^{n} \rightarrow \mathbb{R}$, имеющих компактные в $G$ носители, $C(G)$ - пространство непрерывных 
функций $g: G \rightarrow \mathbb{R}$ с топологией, порожденной семейством полунорм $\|g\|_{L_{\infty}(U)}$, где $U$ пробегает все компактные в $G$ множества, $L_{p}^{k}(G)$ - множество локально суммируемых функций $g: G \rightarrow \mathbb{R}$, у которых все обобщенные производные порядка $k$ принадлежат $L_{p}(G), W_{p}^{k}(G)$ - пространство Соболева с нормой

$$
\|g\|_{W_{p}^{k}(G)}=\max _{|\alpha| \leqslant k}\left\|D^{\alpha} g\right\|_{L_{p}(G)}
$$

где $G$ - открытое множество в $\mathbb{R}^{n}, k \in \mathbb{Z}_{+}, 1 \leqslant p \leqslant \infty$; при $g \in L_{p}^{k}(G), r>0$

$$
\begin{aligned}
\left\|D^{k} g\right\|_{L_{p}(G)} & = \begin{cases}\left(\int_{G} \sum_{|\alpha|=k} \frac{k !}{\alpha !}\left|D^{\alpha} g\right|^{p} d x\right)^{1 / p} \text { при } p<\infty, \\
\max _{|\alpha|=k}\left\|D^{\alpha} g\right\|_{L_{\infty}(G)} & \text { при } p=\infty,\end{cases} \\
\omega\left(D^{k} g, r\right)_{L_{p}(G)} & =\max _{|\alpha|=k} \sup _{|a| \leqslant r}\left\|D^{\alpha} g(x)-D^{\alpha} g(x-a)\right\|_{L_{p}(G \cap(a+G)),} \\
\tilde{\omega}\left(D^{k} g, r\right)_{L_{p}(G)} & =\max _{|\alpha|=k}\left\|D^{\alpha} g(x)-D^{\alpha} g(y)\right\|_{L_{p}\left(F_{r}\right)},
\end{aligned}
$$

где $F_{r}=\{(x, y): x, y \in G,|x-y| \leqslant r\} \subset \mathbb{R}^{2 n}$. Введем в $L_{p}^{k}\left(\mathbb{R}^{n}\right)$ норму

$$
\|g\|_{L_{p}^{k}\left(\mathbb{R}^{n}\right)}=\|g\|_{L_{p}(B(0,1))}+\left\|D^{k} g\right\|_{L_{p}\left(\mathbb{R}^{n}\right)}
$$

(эквивалентные функции отождествляются); тогда $L_{p}^{k}\left(\mathbb{R}^{n}\right)$ - банахово пространство (см., например, $\left[6\right.$, с. 134]). Обозначим через $\stackrel{\circ}{L}_{p}^{k}\left(\mathbb{R}^{n}\right)$ замыкание $\mathscr{D}\left(\mathbb{R}^{n}\right)$ в $L_{p}^{k}\left(\mathbb{R}^{n}\right)$. Поскольку $\mu>n / 2$, оператор вложения $L_{2}^{\mu}\left(\mathbb{R}^{n}\right) \rightarrow C\left(\mathbb{R}^{n}\right)$ непрерывен $[4$, с. 128, 145]. Учитывая теорему продолжения $[5$, с. 214$]$, функции из $W_{2}^{\mu}(\Omega)$ будем считать непрерьвньги на $\bar{\Omega}$.

Теорема 1. Допустим, что $\Delta \in \mathscr{A}_{m / 2}$. Тогда существует единственная функиия $s_{m}(f, \Delta)$ вида (1), интерполирующая заданную функиию $f: \Delta \rightarrow \mathbb{R}$ на $\Delta$. При этом $s_{m}(f, \Delta)$ является единственным решением вариачионной задачи

$$
\min \left\{\left\|D^{\mu} g\right\|_{L_{2}\left(\mathbb{R}^{n}\right)}: g \in \stackrel{\circ}{L}{ }_{2}^{\mu}\left(\mathbb{R}^{n}\right), g(x)=f(x) \forall x \in \Delta\right\} .
$$

ДоКАЗАТЕЛЬСТВО. Положим $p=2 n$ при нечетном $n$ и $p=n$ при четном $n$. Заметим, что

$$
L_{2}^{\mu}\left(\mathbb{R}^{n}\right) \cap L_{p}^{m^{\prime}}\left(\mathbb{R}^{n}\right)=\stackrel{\circ}{L_{2}^{\mu}}\left(\mathbb{R}^{n}\right) .
$$

Действительно, обозначим через $\alpha_{j}$ мультииндекс, у которого $j$-я компонента равна $\mu$, а все остальные компоненты равны 0 , через $X$ - пространство локально суммируемых функций $g: \mathbb{R}^{n} \rightarrow \mathbb{R}$, у которых обобщенные производные $D^{\alpha_{j}} g$ принадлежат $L_{2}\left(\mathbb{R}^{n}\right)$ $(j=\overline{1, n})$, с нормой

$$
\|g\|_{X}=\|g\|_{L_{2}(B(0,1))}+\sum_{j=1}^{n}\left\|D^{\alpha_{j}} g\right\|_{L_{2}\left(\mathbb{R}^{n}\right)}
$$


(см. [4, с. 128]). В [4, с. 223-224] для произвольной функции $g \in X$ определен полином $Q(g)$, не содержаший членов степени $\leqslant m / 2$, такой, что $g-Q(g) \in L_{2}^{\mu}\left(\mathbb{R}^{n}\right) \cap L_{p}^{m^{\prime}}\left(\mathbb{R}^{n}\right)$ (очевидно, этими условиями $Q(g)$ определяется однозначно). Положим $Y=\{g \in X$ : $Q(g)=0\}$. Ясно, что $Y=L_{2}^{\mu}\left(\mathbb{R}^{n}\right) \cap L_{p}^{m^{\prime}}\left(\mathbb{R}^{n}\right)$. Нормы $\|\cdot\|_{L_{2}^{\mu}\left(\mathbb{R}^{n}\right)}$ и $\|\cdot\|_{\text {Х эквива- }}$ лентны на $Y[4$, с. 224$]$, а значит, и на $\mathscr{D}\left(\mathbb{R}^{n}\right)$, поэтому $\stackrel{\circ}{L}_{2}^{\mu}\left(\mathbb{R}^{n}\right)$ совпадает с замьканием $\mathscr{D}\left(\mathbb{R}^{n}\right)$ в $X$, которое согласно [4, с. 226] совпадает с $Y$, и мы получаем (4). Минимизирующий элемент в (3) сушествует и единственньй, если не существует ненулевого полинома $P \in \stackrel{\circ}{L}_{2}^{\mu}\left(\mathbb{R}^{n}\right)$, равного 0 на $\Delta[6$, с. $136-137]$. Это условие вьполнено, так как ввиду (4) любой полином, принадлежащий $\stackrel{\circ}{L}_{2}^{\mu}\left(\mathbb{R}^{n}\right)$, имеет степень $\leqslant m / 2$ по совокупности переменных. Обозначим решение задачи (3) через $u$. Для любого $\varphi \in \stackrel{\circ}{L}{ }_{2}^{\mu}\left(\mathbb{R}^{n}\right)$ имеем

$$
\int_{\mathbb{R}^{n}} \sum_{|\alpha|=\mu} \frac{\mu !}{\alpha !} D^{\alpha} u D^{\alpha} \varphi d x=\sum_{j=1}^{N} \xi_{j} \varphi\left(x_{j}\right),
$$

где $\xi_{j} \in \mathbb{R}$ не зависят от $\varphi$ (так как по определению $u$ левая часть (5) равна 0 , если $\varphi \in \stackrel{\circ}{L}_{2}^{\mu}\left(\mathbb{R}^{n}\right)$ и $\left.\varphi\left(x_{j}\right)=0, j=\overline{1, N}\right)$. В силу $(4),(5)$

$$
\sum_{j=1}^{N} \xi_{j} Q\left(x_{j}\right)=0 \quad \forall Q \in \mathscr{P}_{m / 2}
$$

Далее, в $\left[7\right.$, с. 113-114] показано, что если $\varphi \in \mathscr{D}\left(\mathbb{R}^{n}\right)$ и $D^{\alpha} \varphi(0)=0$ при $|\alpha| \leqslant m$, то

$$
\int_{\mathbb{R}^{n}} K \cdot \mathscr{F} \varphi d x=A_{1} \int_{\mathbb{R}^{n}}|x|^{-m-n} \varphi(x) d x,
$$

где $A_{1}=A_{1}(m, n)$ - ненулевая константа. Обозначим функцию

$$
\sum_{j=1}^{N} \xi_{j} K\left(x-x_{j}\right)
$$

через $v(x)$. Используя (7) и учитьвая, что на основании (6) все производные функции $\sum_{j=1}^{N} \xi_{j} e^{-i x x_{j}}$ порядка $\leqslant m / 2$ в точке $x=0$ равны 0 , для любого мультииндекса $\alpha$ с $[(m+1) / 2] \leqslant|\alpha|<m+n$ и любой функции $\psi \in \mathscr{D}\left(\mathbb{R}^{n}\right)$ имеем

$$
\begin{aligned}
\int_{\mathbb{R}^{n}} D^{\alpha} v \cdot \mathscr{F} \psi d x & =\int_{\mathbb{R}^{n}} K(x) \cdot \mathscr{F}\left(\sum_{j=1}^{N} \xi_{j} e^{-i x x_{j}}(i x)^{\alpha} \psi(x)\right) d x \\
& =A_{1} \int_{\mathbb{R}^{n}}\left(\sum_{j=1}^{N} \xi_{j} e^{-i x x_{j}}\right)(i x)^{\alpha}|x|^{-m-n} \psi(x) d x
\end{aligned}
$$

поэтому при $|\alpha|=\mu\left|\mathscr{F} D^{\alpha} v\right| \in L_{2}\left(\mathbb{R}^{n}\right)$ и при $|\alpha|=m^{\prime}\left|\mathscr{F} D^{\alpha} v\right| \in L_{q}\left(\mathbb{R}^{n}\right)$, где $q=p /(p-1)$. Отсюда получаем $v \in L_{2}^{\mu}\left(\mathbb{R}^{n}\right) \cap L_{p}^{m^{\prime}}\left(\mathbb{R}^{n}\right)[8$, c. 201] и согласно (4) 
$v \in \stackrel{\circ}{L}{ }_{2}^{\mu}\left(\mathbb{R}^{n}\right)$. Функция $K(x-y)$ совпадает, с точностью до постоянного множителя, с фундаментальным решением уравнения $\mathscr{L}^{\mu} g(x)=0\left[9\right.$, c. 520-521], так что при $\varphi \in \mathscr{D}\left(\mathbb{R}^{n}\right)$

$$
\int_{\mathbb{R}^{n}} \sum_{|\alpha|=\mu} \frac{\mu !}{\alpha !} D^{\alpha} v D^{\alpha} \varphi d x=(-1)^{\mu} \int_{\mathbb{R}^{n}} \varphi \cdot \mathscr{L}^{\mu} v d x=A_{2} \sum_{j=1}^{N} \xi_{j} \varphi\left(x_{j}\right),
$$

где $A_{2}=A_{2}(m, n)$ - ненулевая константа. Следовательно, при $\varphi \in \stackrel{\circ}{L}{ }_{2}^{\mu}\left(\mathbb{R}^{n}\right)$

$$
\int_{\mathbb{R}^{n}} \sum_{|\alpha|=\mu} \frac{\mu !}{\alpha !} D^{\alpha}\left(A_{2}^{-1} v\right) D^{\alpha} \varphi d x=\sum_{j=1}^{N} \xi_{j} \varphi\left(x_{j}\right)
$$

Вычитая (9) из (5), полагая $\varphi=u-A_{2}^{-1} v$ и учитывая (4), получаем $u-A_{2}^{-1} v \in \mathscr{P}_{m / 2}$. Нам остается показать, что не могут существовать две различные функции вида (1), совпадающие на $\Delta$. Предположим, что $s(x)$ имеет вид $(1)$ и $s(x)=0$ на $\Delta$. Тогда по доказанному $s \in \stackrel{\circ}{L}{ }_{2}^{\mu}\left(\mathbb{R}^{n}\right)$ и

$$
\left\|D^{\mu} s\right\|_{L_{2}\left(\mathbb{R}^{n}\right)}^{2}=\int_{\mathbb{R}^{n}} \sum_{|\alpha|=\mu} \frac{\mu !}{\alpha !} D^{\alpha}\left(\sum_{j=1}^{N} \lambda_{j} K\left(x-x_{j}\right)\right) D^{\alpha} s(x) d x=A_{2} \sum_{j=1}^{N} \lambda_{j} s\left(x_{j}\right)=0 .
$$

Отсюда, из (4) и из условия $\Delta \in \mathscr{A}_{m / 2}$ заключаем, что $s(x) \equiv 0$.

ЗАмЕчАниЕ 1.1. Из рассуждений, приведенных выше для обоснования равенства (4), вытекает также (известное) равенство $L_{2}^{\mu}\left(\mathbb{R}^{n}\right)=\stackrel{\circ}{L}_{2}^{\mu}\left(\mathbb{R}^{n}\right)+\mathscr{P}_{\mu-1}$.

ЗАмЕчание 1.2. Если $\xi_{1}, \ldots, \xi_{N} \in \mathbb{R}$ и $\sum_{j=1}^{N} \xi_{j} Q\left(x_{j}\right) \neq 0$ для некоторого $Q \in \mathscr{P}_{m / 2}$, TO

$$
\sum_{j=1}^{N} \xi_{j} K\left(x-x_{j}\right) \notin L_{2}^{\mu}\left(\mathbb{R}^{n}\right)
$$

Это следует из того, что равенства (8) справедливы в случае, когда

$$
v(x)=\sum_{j=1}^{N} \xi_{j} K\left(x-x_{j}\right)
$$

$|\alpha|=\mu, \psi \in \mathscr{D}\left(\mathbb{R}^{n}\right), D^{\beta} \psi(0)=0$ при $|\beta| \leqslant m$ (не все производные функции

$$
\eta(x)=\sum_{j=1}^{N} \xi_{j} e^{-i x x_{j}}
$$

порядка $\leqslant m / 2$ в точке $x=0$ равны 0 , поэтому $\left.|\eta(x)| x^{\alpha}|x|^{-m-n} \notin L_{2}\left(\mathbb{R}^{n}\right)\right)$.

ЗАмЕчаниЕ 1.3. Факт существования и единственности функции вида (1), интерполирующей заданную функцию $f$ на множестве $\Delta \in \mathscr{A}_{m / 2}$, другим способом установлен в [3]. Но второе утверждение теоремы 1 является новым. 
ТЕОрема 2. Предположим, что функиия $f \in W_{p}^{k}(\Omega)$ непрерывна на $\bar{\Omega}, \Delta \subset \bar{\Omega}$, $h=\bar{h}(\Delta), \varepsilon>0, \varkappa>0$ и выполняются вложения

1) $W_{p}^{k}(\Omega) \rightarrow L_{\infty}(\Omega) u W_{p}^{k}(\Omega) \rightarrow W_{q}^{l}(\Omega), l-n / q<m, \underline{h}(\Delta)>\varkappa h, л u б o$

2) $W_{p}^{k}(\Omega) \rightarrow W_{2}^{\mu}(\Omega) \rightarrow W_{q}^{l}(\Omega)$.

Если $h<C_{0}$, то справедливы неравенства

$$
\begin{aligned}
& \left\|D^{l}\left(f-s_{m}(f, \Delta)\right)\right\|_{L_{q}\left(\Omega_{\varepsilon}\right)} \leqslant C_{1}\left(h^{\theta} \omega\left(D^{k} f, h\right)_{L_{p}(\Omega)}+C_{2}^{1 / h}\|f\|_{L_{1}(\Omega)}\right) \quad n p u k<m+n, \\
& \left\|D^{l}\left(f-s_{m}(f, \Delta)\right)\right\|_{L_{q}\left(\Omega_{\varepsilon}\right)} \leqslant C_{1}\left(h^{\theta}\left\|D^{k} f\right\|_{L_{p}(\Omega)}+C_{2}^{1 / h}\|f\|_{L_{1}(\Omega)}\right) \quad n p u k=m+n,
\end{aligned}
$$

где $\theta=k-l+n / q-n / p$ при $p \leqslant q u \theta=k-l$ nри $p>q$. Здесь $C_{0}=C_{0}(\Omega, m)$, $C_{j}=C_{j}(\Omega, m, p, q, \varepsilon, \varkappa)(j=1,2)$ - положительные константы, $C_{2}<1$.

Нам потребуются следующие утверждения.

ЛЕмма 1. Если $\zeta \geqslant 1, g \in W_{p}^{k}(\zeta \Omega)$ и $а \in \zeta \Omega$, то существуют такие полиномь $P_{1} \in \mathscr{P}_{k}$ и $P_{2} \in \mathscr{P}_{k-1}$, что для любого $r \geqslant 1$

$$
\begin{gathered}
\left\|g-P_{1}\right\|_{W_{p}^{k}\left(B_{\zeta}(a, r)\right)} \leqslant C_{3} r^{C_{4}} \tilde{\omega}\left(D^{k} g, C_{6}\right)_{L_{p}\left(B_{\zeta}\left(a, C_{5} r\right)\right)} \\
\left\|g-P_{2}\right\|_{W_{p}^{k}\left(B_{\zeta}(a, r)\right)} \leqslant C_{3} r^{C_{4}}\left\|D^{k} g\right\|_{L_{p}\left(B_{\zeta}\left(a, C_{5} r\right)\right)}
\end{gathered}
$$

əде $C_{j}=C_{j}(\Omega, k)(j=3,4,5,6)$.

ДокАЗАтЕльство. Утверждение леммы установлено в [6, лемма 3$]$ с той разнищей, что в левых частях неравенств вместо $B_{\zeta}(a, r)$ стоит связная компонента $B_{\zeta}(a, r)$, содержащая $a$. Нужно только заметить, что при наших условиях две различные точки $x, y \in \Omega$ можно соединить ломаной, содержащейся в $\Omega \cap B(x, A|x-y|)$, где $A=A(\Omega)$ (в [6] допускаются неограниченные области $\Omega$ ).

ЛЕмма 2. Пусть $l \in \mathbb{Z}_{+}, 1 \leqslant q \leqslant \infty, l-n / q<m, \zeta>0, \zeta \underline{h}(\Delta)>\rho>0$,

$$
u(x)=\sum_{j=1}^{N} \lambda_{j} K\left(x-x_{j}\right)+P(x)
$$

əде $\lambda_{j} \in \mathbb{R} u P \in \mathscr{P}_{2 \mu-1}, \tilde{u}(x)=u(x / \zeta), a \in \mathbb{R}^{n}$. Тогда $\tilde{u} \in W_{q}^{l}(B(a, 1)) u$

$$
\|\tilde{u}\|_{W_{q}^{l}(B(a, 1))} \leqslant C_{7}\|\tilde{u}\|_{L_{1}(B(a, 2))}
$$

əде $C_{7}=C_{7}(m, n, q, \rho)$. 
ДокаЗАТЕЛЬСТво. Функция $K(x)$ - полигармоническая порядка $\mu$ на $\mathbb{R}^{n} \backslash\{0\}[9$, c. 520-521] и $K \in W_{q}^{l}(B(0,1))$. Далее, если $g$ - некоторая полигармоническая функция порядка $\mu$ на шаре $B(0, r)$, то для любого мультииндекса $\alpha$

$$
\left|D^{\alpha} g(0)\right| \leqslant A_{1}\|g\|_{L_{1}(B(0, r))},
$$

где $A_{1}=A_{1}(m, n, r, \alpha)$ (это вытекает из существования функции $\varphi \in \mathscr{D}(B(0, r / 2))$, зависящей только от $m, n, r$ такой, что $g=g * \varphi$ на $B(0, r / 2)[10, c .132-135])$. Поэтому достаточно установить, что если $\mathscr{L}^{\mu} g(x)=0$ на $B(0,2)$ и $\lambda \in \mathbb{R}$, то

$$
\|\lambda K+g\|_{W_{q}^{l}(B(0,1))} \leqslant A_{2}\|\lambda K+g\|_{L_{1}(B(0,2))},
$$

где $A_{2}=A_{2}(m, n, q)$. Если это неверно, то существуют такие $g_{j}(x)$ и $\lambda_{j} \in \mathbb{R}(j \in \mathbb{N})$, что $\mathscr{L}^{\mu} g_{j}(x)=0$ на $B(0,2)$,

$$
\lim _{j \rightarrow \infty}\left\|\lambda_{j} K+g_{j}\right\|_{W_{q}^{l}(B(0,1))}=\infty, \quad \lim _{j \rightarrow \infty}\left\|\lambda_{j} K+g_{j}\right\|_{L_{1}(B(0,2))}=0
$$

В случае

$$
\varliminf_{j \rightarrow \infty}\left|\lambda_{j}\right|=0
$$

получаем противоречие с (12), а в противном случае имеем

$$
\lim _{j \rightarrow \infty}\left\|K+\lambda_{j}^{-1} g_{j}\right\|_{L_{1}(B(0,2))}=0
$$

откуда в силу (12) следует бесконечная дифференцируемость $K(x)$ на $B(0,2)$, что неверно.

Лемма 3 [6, лемма 5]. Допустим, что $\zeta>0, F \subset \zeta \Omega, \Phi \subset W_{2}^{\mu}(\zeta \Omega), 0<r_{0}<C_{8} \zeta$ и выполнены условия:

1) для любых $g \in \Phi, x \in \zeta \Omega$ найдется такое $y \in \zeta \bar{\Omega}$, ито $g(y)=0,|x-y| \leqslant r_{0}$;

2) если $\varphi \in W_{\infty}^{\mu}(\zeta \Omega), \varphi$ бесконечно дифферениируема на $\zeta \Omega$ и $\varphi(x)=1 \mathrm{nри}$ $x \in F$, то $g \varphi \in \Phi$ для любого $g \in \Phi$;

3) существует такое $u \in \Phi$, ито $\left\|D^{\mu} u\right\|_{L_{2}(\zeta \Omega)}=\inf _{g \in \Phi}\left\|D^{\mu} g\right\|_{L_{2}(\zeta \Omega)}$.

Тогда для любого $r \in \mathbb{R}$

$$
\|u\|_{W_{2}^{\mu}\left(V_{r}\right)} \leqslant C_{9} C_{10}^{r / r_{0}}\left(1+r_{0}\right)^{\mu}\left\|D^{\mu} u\right\|_{L_{2}(\zeta \Omega)},
$$

əде $V_{r}=\{x \in \zeta \Omega: \operatorname{dist}(x, F)>r\}$. Здесь $C_{j}=C_{j}(\Omega, m)$ - положительные константы $(j=8,9,10), C_{10}<1$. 
ДокАЗАТЕЛЬСТво тЕоремЫ 2. Предположим сначала, что $k<m+n$. Зафиксируем некоторую функцию $\varphi=\varphi_{n} \in \mathscr{D}(B(0,1))$, удовлетворяющую условиям: $\varphi(x) \geqslant 0$ при $x \in \mathbb{R}^{n}, \varphi(x)=1$ при $|x| \leqslant 1 / 3, \int_{\mathbb{R}^{n}} \varphi d x=1$. Положим

$$
\psi(x)=\varphi(x)\left(\sum_{y \in \frac{1}{2 n} \mathbb{Z}^{n}} \varphi(x+y)\right)^{-1} .
$$

Будем считать, что $h<\min \left(d / 4, C_{8} / 6\right)$, где $d$ - диаметр $\Omega, C_{8}$ - константа из леммы 3 и $(U \subset \bar{\Omega}, U \neq \varnothing, \bar{h}(U)=h) \Longrightarrow\left(U \in \mathscr{A}_{m / 2}\right)$. Заметим, что для любого $y \in \mathbb{R}^{n}$

$$
\sup _{x \in \Omega} \inf _{x^{\prime} \in \Delta \backslash B(y, h)}\left|x-x^{\prime}\right| \leqslant 6 h .
$$

Действительно, если $x \in \Omega \backslash B(y, 2 h)$, то для некоторого $x^{\prime} \in \Delta\left|x-x^{\prime}\right| \leqslant h$, и тогда $x^{\prime} \in \Delta \backslash B(y, h)$. Если же $x \in \Omega \cap B(y, 2 h)$, то ввиду $d>4 h$ найдется точка $a \in \Omega \cap B(y, 3 h) \backslash B(y, 2 h)$; для некоторого $x^{\prime} \in \Delta$ имеем $\left|a-x^{\prime}\right| \leqslant h$, следовательно, $x^{\prime} \in \Delta \backslash B(y, h)$ и

$$
\left|x-x^{\prime}\right| \leqslant|x-y|+|y-a|+\left|a-x^{\prime}\right| \leqslant 2 h+3 h+h \leqslant 6 h .
$$

Ниже $A_{j}$ - некоторые положительные константы, зависящие от $\Omega, m, p, q, \varepsilon, \varkappa$. Продолжим функцию $f$ на $\mathbb{R}^{n}$ так, чтобы $f \in W_{p}^{k}\left(\mathbb{R}^{n}\right)$,

$$
\|f\|_{W_{p}^{k}\left(\mathbb{R}^{n}\right)} \leqslant A_{1}\|f\|_{W_{p}^{k}(\Omega)}
$$

(см. [5, с. 214]), и положим $\zeta=\max \left(1,3 / \varepsilon, 1 / h, C_{6} / h\right)$, где $C_{6}-$ константа из леммы 1 , $s=s_{m}(f, \Delta), \tilde{f}(x)=f(x / \zeta), \tilde{s}(x)=s(x / \zeta)$.

Обозначим через $E$ множество $\left\{x \in \frac{1}{n} \mathbb{Z}^{n}: \operatorname{dist}\left(x, \zeta \Omega_{\varepsilon}\right) \leqslant 1\right\}$ и возьмем некоторую точку $z \in E$; ввиду $\zeta \varepsilon \geqslant 3$

$$
B(z, 2) \subset \zeta \Omega \text {. }
$$

Пусть $P_{1}$ - полином из леммы 1 , соответствующий функции $g=\tilde{f}$ при $a=z(\zeta \geqslant 1)$, и $P=\chi P_{1}$, где $\chi$ есть свертка $\varphi$ с характеристической функцией множества $\left\{x \in \mathbb{R}^{n}\right.$ : $\operatorname{dist}(x, \zeta \Omega) \leqslant 1\}$. Как легко видеть,

$$
\left\|P_{1}\right\|_{W_{p}^{k}(B(z, 1))} \leqslant A_{2}\|\tilde{f}\|_{W_{p}^{k}\left(B\left(z, A_{3}\right)\right)} .
$$

Введем обозначения

$$
\begin{gathered}
\pi_{y}=\operatorname{dist}\left(B(z, 2), B_{\zeta}(y, 1) \cup \zeta\left(\Omega \backslash \Omega_{\varepsilon / 2}\right)\right), \\
f_{y}(x)=(\tilde{f}(x)-P(x)) \psi(x-y), \\
s_{y}=s_{m}\left(f_{y}, \zeta \Delta\right), \quad v=P-s_{m}(P, \zeta \Delta),
\end{gathered}
$$

где $y \in \mathbb{R}^{n}$. Сейчас с помощью леммы 3 мы установим неравенства

$$
\begin{gathered}
\left\|s_{y}\right\|_{W_{2}^{\mu}(B(z, 2))} \leqslant A_{4} A_{5}^{\pi_{y}}\left\|D^{\mu} s_{y}\right\|_{L_{2}(\zeta \Omega)}, \\
\|v\|_{W_{2}^{\mu}(B(z, 2))} \leqslant A_{4} A_{5}^{1 / h}\left\|D^{\mu} v\right\|_{L_{2}(\zeta \Omega)},
\end{gathered}
$$


где $A_{5}<1$. Из теоремы 1 вытекает, что $s_{y}$ является решением вариационной задачи

$$
\min \left\{\left\|D^{\mu} g\right\|_{L_{2}(\zeta \Omega)}: g \in \Phi\right\}
$$

где $\Phi=\left\{g \in W_{2}^{\mu}(\zeta \Omega): g(x)=s_{y}(x) \forall x \in \zeta\left(\Delta \cup\left(\Omega \backslash \Omega_{\varepsilon / 2}\right)\right)\right\}$. Поскольку любая функция $g \in \Phi$ равна 0 на $(\zeta \Delta) \backslash B(y, 1)$, в силу (13) имеем

$$
\sup _{x \in \zeta \Omega} \inf _{x^{\prime} \in(\zeta \Delta) \backslash B(y, 1)}\left|x-x^{\prime}\right| \leqslant 6 \zeta h .
$$

Применяя лемму 3 с $F=B_{\zeta}(y, 1) \cup \zeta\left(\Omega \backslash \Omega_{\varepsilon / 2}\right), r_{0}=6 \zeta h\left(r_{0}<C_{8} \zeta\right.$, так как $\left.h<C_{8} / 6\right)$, $u=s_{y}$, получаем (17). Аналогичным образом можно применить лемму 3 и к функции $v$, положив $\Phi=\left\{g \in W_{2}^{\mu}(\zeta \Omega): g(x)=v(x) \forall x \in \zeta\left(\Delta \cup\left(\Omega \backslash \Omega_{\varepsilon / 2}\right)\right)\right\}, F=\zeta\left(\Omega \backslash \Omega_{\varepsilon / 2}\right)$, $r_{0}=\zeta h$. Нужно только учесть, что

a) $v$ является решением вариационной задачи вида (19) при указанном здесь $\Phi$;

б) в силу $z \in E$ выполнено $\operatorname{dist}\left(B(z, 2), \zeta\left(\Omega \backslash \Omega_{\varepsilon / 2}\right)\right) \geqslant A_{6} h^{-1}-A_{7}$.

Для проверки утверждения а) заметим, что оно эквивалентно равенству

$$
\int_{\zeta \Omega} \sum_{|\alpha|=\mu} \frac{\mu !}{\alpha !} D^{\alpha} v D^{\alpha} g d x=0
$$

выполняющемуся для любой функции $g \in W_{2}^{\mu}(\zeta \Omega)$, равной 0 на $\zeta\left(\Delta \cup\left(\Omega \backslash \Omega_{\varepsilon / 2}\right)\right)$. Поскольку $P(x)=P_{1}(x)$ при $x \in \zeta \Omega, P_{1} \in \mathscr{P}_{2 \mu-1}$, имеем

$$
\int_{\zeta \Omega} \sum_{|\alpha|=\mu} \frac{\mu !}{\alpha !} D^{\alpha} P D^{\alpha} g d x=(-1)^{\mu} \int_{\zeta \Omega} \mathscr{L}^{\mu} P \cdot g d x=0 .
$$

Соотношение вида (20) с заменой $v$ на $s_{m}(P, \zeta \Delta)$ справедливо ввиду соответствующего экстремального свойства функции $s_{m}(P, \zeta \Delta)$ (теорема 1 ). Таким образом, неравенство (18) доказано.

Далее, если имеет место случай 1 ) в теореме 2 , то, используя лемму 2 , неравенство $\zeta \underline{h}(\Delta)>\varkappa$ и $(15)$, запишем

$$
\begin{aligned}
\left\|s_{y}\right\|_{W_{q}^{l}(B(z, 1))} & \leqslant A_{8}\left\|s_{y}\right\|_{W_{2}^{\mu}(B(z, 2))} \\
\|v\|_{W_{q}^{l}(B(z, 1))} & \leqslant A_{8}\|v\|_{W_{2}^{\mu}(B(z, 2))}
\end{aligned}
$$

Кроме того, применяя теорему 1 и учитывая вложение $W_{p}^{k}(B(0,1)) \rightarrow L_{\infty}(B(0,1))$, которое выполняется в силу теоремы продолжения [5, с. 214], получаем

$$
\begin{aligned}
\left\|D^{\mu} s_{y}\right\|_{L_{2}\left(\mathbb{R}^{n}\right)} & \leqslant\left\|D^{\mu}\left(\sum_{a \in \zeta \Delta} f_{y}(a) \varphi\left(\frac{x-a}{\varkappa}\right)\right)\right\|_{L_{2}\left(\mathbb{R}^{n}\right)} \leqslant A_{9}\left\|f_{y}\right\|_{L_{\infty}(B(y, 1))} \\
& \leqslant A_{10}\left\|f_{y}\right\|_{W_{p}^{k}(B(y, 1))},
\end{aligned}
$$

откуда

$$
\left\|D^{\mu} s_{y}\right\|_{L_{2}\left(\mathbb{R}^{n}\right)} \leqslant A_{11}\|\tilde{f}-P\|_{W_{p}^{k}(B(y, 1))} .
$$


В случае 2) неравенства (21), (22) справедливы согласно вложению $W_{2}^{\mu}(B(0,1)) \rightarrow$ $W_{q}^{l}(B(0,1))$, а для того чтобы получить $(23)$, необходимо оценить $\left\|D^{\mu} s_{y}\right\|_{L_{2}\left(\mathbb{R}^{n}\right)}$ через $\left\|D^{\mu} f_{y}\right\|_{L_{2}\left(\mathbb{R}^{n}\right)}$ и использовать вложение $W_{p}^{k}(B(0,1)) \rightarrow W_{2}^{\mu}(B(0,1))$. На основании (14), (16)

$$
\max \left(\|\tilde{f}\|_{W_{p}^{k}\left(\mathbb{R}^{n}\right)},\|P\|_{W_{p}^{k}\left(\mathbb{R}^{n}\right)},\|P\|_{W_{2}^{\mu}\left(\mathbb{R}^{n}\right)}\right) \leqslant A_{12} h^{-A_{13}}\|f\|_{W_{p}^{k}(\Omega)} .
$$

Пусть $Y_{1}\left(Y_{2}\right)$ - множество точек $y \in \frac{1}{2 n} \mathbb{Z}^{n}$ таких, что $|y-z|<\zeta \varepsilon-2$ (соответственно $\left.|y-z| \geqslant \zeta \varepsilon-2, B_{\zeta}(y, 1) \neq \varnothing\right)$, и $Y=Y_{1} \cup Y_{2}$. Из условия $z \in E$ следует, что при $y \in Y_{1}$ $\pi_{y} \geqslant A_{14}|y-z|-A_{15}$ и $B(y, 1) \subset \zeta \Omega$, а при $y \in Y_{2} \pi_{y} \geqslant A_{16} h^{-1}-A_{17}$. Учитьвая это и обозначая $r_{y z}=|y-z|+1$, согласно $(17),(21),(23),(24)$ имеем

$$
\begin{aligned}
\left\|s_{y}\right\|_{W_{q}^{l}(B(z, 1))} & \leqslant A_{18} A_{19}^{r_{y z}}\|\tilde{f}-P\|_{W_{p}^{k}(B(y, 1))} \\
& \leqslant A_{20} A_{21}^{r_{y z}} \tilde{\omega}\left(D^{k} \tilde{f}, C_{6}\right)_{L_{p}\left(B_{\zeta}\left(z, A_{22} r_{y z}\right)\right)} \quad \forall y \in Y_{1},
\end{aligned}
$$

где $A_{19}, A_{21}<1$, и

$$
\left\|s_{y}\right\|_{W_{q}^{l}(B(z, 1))} \leqslant A_{23} A_{24}^{1 / h}\|\tilde{f}-P\|_{W_{p}^{k}(B(y, 1))} \leqslant A_{25} A_{26}^{1 / h}\|f\|_{W_{p}^{k}(\Omega)} \quad \forall y \in Y_{2},
$$

где $A_{24}, A_{26}<1$. В силу $(18),(22),(24)$ и теоремы 1

$$
\|v\|_{W_{q}^{l}(B(z, 1))} \leqslant A_{27} A_{28}^{1 / h}\left\|D^{\mu} v\right\|_{L_{2}\left(\mathbb{R}^{n}\right)} \leqslant 2 A_{27} A_{28}^{1 / h}\left\|D^{\mu} P\right\|_{L_{2}\left(\mathbb{R}^{n}\right)} \leqslant A_{29} A_{30}^{1 / h}\|f\|_{W_{p}^{k}(\Omega)},
$$

где $A_{28}, A_{30}<1$. Рассматриваемьй метод интерполирования является линейным и коммутирует с преобразованием подобия (по теореме 1 ), так что

$$
\tilde{f}-\tilde{s}=\tilde{f}-P-\sum_{y \in Y} s_{y}+v .
$$

Обозначим $\tau_{a r}=\tilde{\omega}\left(D^{k} \tilde{f}, C_{6}\right)_{L_{p}\left(B_{\zeta}(a, r)\right)}$. Теперь, используя вложение $W_{p}^{k}(B(0,1)) \rightarrow$ $W_{q}^{l}(B(0,1)),(15)$ и то, что $Y_{2}$ содержит $\leqslant A_{31} h^{-n}$ точек, из $(25)-(28)$ получаем

$$
\begin{aligned}
\|\tilde{f}-\tilde{s}\|_{W_{q}^{l}(B(z, 1))} \leqslant & \|\tilde{f}-P\|_{W_{q}^{l}(B(z, 1))}+\sum_{y \in Y}\left\|s_{y}\right\|_{W_{q}^{l}(B(z, 1))}+\|v\|_{W_{q}^{l}(B(z, 1))} \\
\leqslant & A_{32}\|\tilde{f}-P\|_{W_{p}^{k}(B(z, 1))}+\sum_{y \in Y_{1}} A_{20} A_{21}^{r_{y z}} \tilde{\omega}\left(D^{k} \tilde{f}, C_{6}\right)_{L_{p}\left(B_{\zeta}\left(z, A_{22} r_{y z}\right)\right)} \\
& +\sum_{y \in Y_{2}} A_{25} A_{26}^{1 / h}\|f\|_{W_{p}^{k}(\Omega)}+A_{29} A_{30}^{1 / h}\|f\|_{W_{p}^{k}(\Omega)} \\
\leqslant & A_{33}\left(\tau_{z A_{34}}+\sum_{j=1}^{\infty} A_{35}^{j} \tau_{z j}+A_{36}^{1 / h}\|f\|_{W_{p}^{k}(\Omega)}\right) \\
\leqslant & A_{37}\left(\sum_{j=1}^{\infty} A_{35}^{j} \tau_{z j}+A_{36}^{1 / h}\|f\|_{W_{p}^{k}(\Omega)}\right)
\end{aligned}
$$


где $A_{35}, A_{36}<1$.

Положим $i=0$ при $p \leqslant q, i=1$ при $p>q$ и обозначим через $\xi_{r}(x, y)$ количество точек $z \in E$ таких, что $|x-z|<r,|y-z|<r$. Ввиду того, что $E$ содержит $\leqslant A_{38} h^{-n}$ точек и $\xi_{r}(x, y) \leqslant A_{39} r^{n}$ при $r \geqslant 1$, имеем

$$
\begin{aligned}
& \left(\sum_{z \in E}\left(\sum_{j=1}^{\infty} A_{35}^{j} \tau_{z j}\right)^{q}\right)^{1 / q} \leqslant A_{40} h^{i(n / p-n / q)}\left(\sum_{z \in E}\left(\sum_{j=1}^{\infty} A_{35}^{j} \tau_{z j}\right)^{p}\right)^{1 / p} \\
& \leqslant A_{41} h^{i(n / p-n / q)}\left(\sum_{z \in E} \sum_{j=1}^{\infty} A_{35}^{j} \tau_{z j}^{p}\right)^{1 / p} \\
& \leqslant A_{41} h^{i(n / p-n / q)}\left(\sum_{z \in E} \sum_{j=1}^{\infty} \sum_{|\alpha|=k} A_{35}^{j} \iint_{x, y \in B_{\zeta}(z, j)}\left|D^{\alpha} \tilde{f}(x)-D^{\alpha} \tilde{f}(y)\right|^{p} d x d y\right)^{1 / p} \\
& =A_{41} h^{i(n / p-n / q)}\left(\sum_{|\alpha|=k} \iint_{\mid x-y \in \zeta \Omega}^{x, y \in C_{6}}\left|D^{\alpha} \tilde{f}(x)-D^{\alpha} \tilde{f}(y)\right|^{p}\left(\sum_{j=1}^{\infty} A_{35}^{j} \xi_{j}(x, y)\right) d x d y\right)^{1 / p} \\
& \leqslant A_{42} h^{i(n / p-n / q)} \tilde{\omega}\left(D^{k} \tilde{f}, C_{6}\right)_{L_{p}(\zeta \Omega)} \leqslant A_{43} h^{i(n / p-n / q)} \omega\left(D^{k} \tilde{f}, C_{6}\right)_{L_{p}(\zeta \Omega)}
\end{aligned}
$$

(считаем, что $p, q<\infty$, но это ограничение несущественно). Из (29), (30) получаем

$$
\begin{aligned}
\left\|D^{l}(\tilde{f}-\tilde{s})\right\|_{L_{q}\left(\zeta \Omega_{\varepsilon}\right)} & \leqslant\left(\sum_{z \in E}\left\|D^{l}(\tilde{f}-\tilde{s})\right\|_{L_{q}(B(z, 1))}^{q}\right)^{1 / q} \\
& \leqslant A_{44}\left(\sum_{z \in E}\left(\sum_{j=1}^{\infty} A_{35}^{j} \tau_{z j}+A_{36}^{1 / h}\|f\|_{W_{p}^{k}(\Omega)}\right)^{q}\right)^{1 / q} \\
& \leqslant A_{44}\left(\sum_{z \in E}\left(\sum_{j=1}^{\infty} A_{35}^{j} \tau_{z j}\right)^{q}\right)^{1 / q}+A_{44}\left(\sum_{z \in E}\left(A_{36}^{1 / h}\|f\|_{W_{p}^{k}(\Omega)}\right)^{q}\right)^{1 / q} \\
& \leqslant A_{45}\left(h^{i(n / p-n / q)} \omega\left(D^{k} \tilde{f}, C_{6}\right)_{L_{p}(\zeta \Omega)}+A_{46}^{1 / h}\|f\|_{W_{p}^{k}(\Omega)}\right)
\end{aligned}
$$

где $A_{46}<1$. Применяя преобразование подобия, находим, что

$$
\left\|D^{l}(f-s)\right\|_{L_{q}\left(\Omega_{\varepsilon}\right)} \leqslant A_{47}\left(h^{\theta} \omega\left(D^{k} f, h\right)_{L_{p}(\Omega)}+A_{48}^{1 / h}\|f\|_{W_{p}^{k}(\Omega)}\right),
$$

где $A_{48}<1\left(C_{6} / \zeta \leqslant h\right)$. Из леммы 1 следует, что

$$
\inf _{Q \in \mathscr{P}_{k}}\|f-Q\|_{W_{p}^{k}(\Omega)} \leqslant A_{49} h^{-A_{50}} \omega\left(D^{k} f, h\right)_{L_{p}(\Omega)},
$$

откуда вытекает неравенство

$$
\|f\|_{W_{p}^{k}(\Omega)} \leqslant A_{51} h^{-A_{50}}\left(\omega\left(D^{k} f, h\right)_{L_{p}(\Omega)}+\|f\|_{L_{1}(\Omega)}\right) .
$$

Наконец, из (31), (33) получаем оценку (10).

В случае $k=m+n$ рассуждения аналогичны. Нужно лишь вместо полинома $P_{1}$ использовать $P_{2}$, когда применяем лемму 1 к функции $\tilde{f}$, и заменить $\omega, \tilde{\omega}$ на соответствуюшие нормы. 
ЗАмечаниЕ 2.1. Теорема 2 останется в силе, если заменить в ней условия $k<m+n$, $k=m+n$ на $k \leqslant m / 2, k=[m / 2]+1$ соответственно и в правых частях неравенств $(10),(11)$ убрать член $C_{2}^{1 / h}\|f\|_{L_{1}(\Omega)}$. Это следует из того, что $s_{m}(P, \Delta)=P$ при $P \in \mathscr{P}_{m / 2}$, $\Delta \in \mathscr{A}_{m / 2}$, а также из неравенства $(32)$ и

$$
\inf _{Q \in \mathscr{P}_{k-1}}\|f-Q\|_{W_{p}^{k}(\Omega)} \leqslant A\left\|D^{k} f\right\|_{L_{p}(\Omega)}
$$

где $A=A(\Omega, k)$ (лемма 1$)$.

ЗАмЕчАнИЕ 2.2. В [3] показано, что если $f \in W_{2}^{\mu}(\Omega)$ и $\Delta_{j}$ - конечное подмножество $\bar{\Omega}(j \in \mathbb{N}), \Delta_{j} \in \mathscr{A}_{m / 2}, \lim _{j \rightarrow \infty} \bar{h}\left(\Delta_{j}\right)=0$, то $s_{m}\left(f, \Delta_{j}\right) \rightarrow f$ в $W_{2}^{\mu}(\Omega)$ при $j \rightarrow \infty$.

ЗАмЕчАниЕ 2.3. Как показано в [11], вариационная задача, получающаяся из (3) заменой $\stackrel{\circ}{L}_{2}^{\mu}\left(\mathbb{R}^{n}\right)$ на $L_{2}^{\mu}\left(\mathbb{R}^{n}\right)$, имеет единственное решение $\sigma_{m}(f, \Delta)$, если $\Delta \in \mathscr{A}_{\mu-1}$, причем

$$
\sigma_{m}(f, \Delta)(x)=\sum_{j=1}^{N} \lambda_{j} K\left(x-x_{j}\right)+P(x),
$$

где $\lambda_{j} \in \mathbb{R}, P \in \mathscr{P}_{\mu-1}$. Функции $\sigma_{m}(f, \Delta)$ обычно называют $D^{\mu}$-сплайнами, но точнее было бы назвать $\sigma_{m}(f, \Delta) D^{\mu}$-сплайном в $L_{2}^{\mu}\left(\mathbb{R}^{n}\right)$, а $s_{m}(f, \Delta)-D^{\mu}$-сплайном в $\stackrel{\circ}{L}_{2}^{\mu}\left(\mathbb{R}^{n}\right)$. Аппроксимативные свойства сплайнов $\sigma_{m}(f, \Delta)$ изучались Ж. Дюшоном [11], [12] и автором [13]. В случае интерполяции сплайнами $\sigma_{m}(f, \Delta)$ проходят рассуждения, по сушеству не отличаюшиеся от доказательства теоремы 2 , так что эта теорема останется справедливой, если заменить в ней $s_{m}(f, \Delta)$ на $\sigma_{m}(f, \Delta)$ (этот результат был анонсирован в [13]).

В заключение отметим связь наших результатов с теорией условно положительно определенных функций, изложенной в работе У. Мэдича, С. Нельсона [14]. Область определения некоторой функции $g$ будем обозначать $X_{g}$. Пусть $M\left(M^{\prime}\right)-$ множество всех функций со значениями в $\mathbb{R}$ (соответственно в $\mathbb{C}$ ), области определения которых являются непустыми конечными подмножествами $\mathbb{R}^{n}$. Для любых $g \in C\left(\mathbb{R}^{n}\right), \nu \in M^{\prime}$ положим

$$
\begin{gathered}
S(g, \nu)(x)=\sum_{y \in X_{\nu}} \nu(y) g(x-y), \quad x \in \mathbb{R}^{n} \\
R_{1}(g, \nu)=\sum_{x \in X_{\nu}} \nu(x) g(x), \quad R_{2}(g, \nu)=\sum_{x, y \in X_{\nu}} \nu(x) \overline{\nu(y)} g(x-y) .
\end{gathered}
$$

Множество функций $\nu \in M\left(\nu \in M^{\prime}\right)$ таких, что $R_{1}(Q, \nu)=0 \forall Q \in \mathscr{P}_{t}$, обозначим через $M_{t}$ (соответственно $\left.M_{t}^{\prime}\right)$. Функция $w \in C\left(\mathbb{R}^{n}\right)$ назьвается условно положительно определенной порядка $t$, если $R_{2}(w, \nu) \geqslant 0 \forall \nu \in M_{t-1}^{\prime}$, где $t \in \mathbb{Z}_{+}$. Обозначим множество всех условно положительно определенных функций порядка $t$ через $\Pi_{t}$. В [14] показано (в [14] рассматриваются комплекснозначные функции, но все рассуждения из этой работы проходят также в вещественном случае, и мы приводим вещественный аналог результатов [14]), что в случае $w \in \Pi_{t}$ существует единственное линейное 
пространство $H_{t}(w) \subset C\left(\mathbb{R}^{n}\right)$ с неотрицательной симметрической билинейной формой $\left(g_{1}, g_{2}\right): H_{t}(w) \times H_{t}(w) \rightarrow \mathbb{R}$, удовлетворяющее условиям:

а) пространство $H_{t}(w)$, снабженное полунормой $\|g\|_{H_{t}(w)}=(g, g)^{1 / 2}$, полно;

б) если $\nu \in M_{t-1}, P \in \mathscr{P}_{t-1}$, то $S(w, \nu)+P \in H_{t}(w)$ и $(S(w, \nu)+P, g)=R_{1}(g, \nu)$ $\forall g \in H_{t}(w)$.

При этом условие $w \in \Pi_{t}$ необходимо для существования пространства $H_{t}(w)$, обладающего указанными свойствами, и в случае $w \in \Pi_{t}$

$$
H_{t}(w)=\left\{g \in C\left(\mathbb{R}^{n}\right): \sup _{\substack{\nu \in M_{t-1} \\ R_{2}(w, \nu) \leqslant 1}} R_{1}(g, \nu)<+\infty\right\}
$$

Кроме того, в [14] показано, что если $w \in \Pi_{t}, \Delta \in \mathscr{A}_{t-1}, f: \Delta \rightarrow \mathbb{R}$ - некоторая функция и допустимое множество вариационной задачи

$$
\min \left\{\|g\|_{H_{t}(w)}: g \in H_{t}(w), g(x)=f(x) \forall x \in \Delta\right\}
$$

непусто, то эта задача имеет единственное решение, которое имеет вид $S(w, \nu)+P$, где $\nu \in M_{t-1}, X_{\nu}=\Delta, P \in \mathscr{P}_{t-1}$.

Далее, пространство, состоящее из тех же функций, что и $L_{2}^{\mu}\left(\mathbb{R}^{n}\right)$, снабженное полунормой $\left\|D^{\mu} g\right\|_{L_{2}\left(\mathbb{R}^{n}\right)}$, полно (это вытекает из неравенства (34) и из полноты фактор-пространства банахова пространства). Ввиду замечания 1.1 пространство $L_{2}^{\mu}\left(\mathbb{R}^{n}\right)$ с полунормой $\left\|D^{\mu} g\right\|_{L_{2}\left(\mathbb{R}^{n}\right)}$ тоже полно. Из доказательства теоремы 1 и из замечания 1.1 следует, что указанное вьше условие б) будет вьполнено, если взять $w(x)=C_{m n} K(x)$, где $C_{m n}$ - ненулевая константа,

$$
\left(g_{1}, g_{2}\right)=\int_{\mathbb{R}^{n}} \sum_{|\alpha|=\mu} \frac{\mu !}{\alpha !} D^{\alpha} g_{1} D^{\alpha} g_{2} d x
$$

и либо $t=m^{\prime}, H_{t}(w)=\stackrel{\circ}{L} \mu\left(\mathbb{R}^{n}\right)$, либо $t=\mu, H_{t}(w)=L_{2}^{\mu}\left(\mathbb{R}^{n}\right)$. Поэтому $C_{m n} K \in \Pi_{m^{\prime}} \subset \Pi_{\mu}$ и $H_{m^{\prime}}\left(C_{m n} K\right)=\stackrel{\circ}{L}{ }_{2}^{\mu}\left(\mathbb{R}^{n}\right), H_{\mu}\left(C_{m n} K\right)=L_{2}^{\mu}\left(\mathbb{R}^{n}\right)$. Таким образом, $C_{m n} K(x)$ можно рассматривать как условно положительно определенную функцию порядка $m^{\prime}$ или порядка $\mu \geqslant m^{\prime}$; в первом случае решением соответствующей вариационной задачи (36) является $D^{\mu}$-сплайн в $\stackrel{\circ}{L}_{2}^{\mu}\left(\mathbb{R}^{n}\right) s_{m}(f, \Delta)$, а во втором случае - $D^{\mu}$-сплайн в $L_{2}^{\mu}\left(\mathbb{R}^{n}\right)$ $\sigma_{m}(f, \Delta)$. Подчеркнем, что $m^{\prime}$ есть наименьшее $t \in \mathbb{Z}_{+}$, при котором $K \in \Pi_{t}$ или $-K \in \Pi_{t}$. Действительно, допустим, что $K \in \Pi_{m^{\prime}-1}$ или $-K \in \Pi_{m^{\prime}-1}$. Если $t_{1}, t_{2} \in \mathbb{Z}_{+}$, $t_{1} \leqslant t_{2}, C \in \mathbb{R} \backslash\{0\}, w \in \Pi_{t_{1}}, C w \in \Pi_{t_{2}}$, то в силу (35) $H_{t_{1}}(w) \subset H_{t_{2}}(C w)$, поэтому для любого $\nu \in M_{m^{\prime}-2} S(K, \nu) \in H_{\mu}\left(C_{m n} K\right)=L_{2}^{\mu}\left(\mathbb{R}^{n}\right)$, а это неверно по замечанию 1.2 . 


\section{СПИСОК ЦИТИРОВАННОЙ ЛИТЕРАТУРЫ}

[1] Алберг Дж., Нильсон Э., Уолш Дж. Теория сплайнов и ее приложения. М.: Мир, 1972.

[2] Шадрин А. Ю. О приближении функций интерполяционными сплайнами, заданными на неравномерных сетках // Матем. сб. 1990. Т. 181. №9. С. 1236-1255.

[3] Duchon J. Splines minimizing rotation-invariant semi-norms in Sobolev spaces // Lecture Notes in Math. 1977. V. 571. P. 85-100.

[4] Бесов О.В., Ильин В. П., Никольский С. М. Интегральные представления функций и теоремы вложения. М.: Наука 1975.

[5] Стейн И. Сингулярные интегралы и дифференциальные свойства функций. М.: Мир, 1973.

[6] Матвеев О.В. Сплайн-интерполяция функций нескольких переменных и базисы в пространствах Соболева // Тр. МИРАН. 1992. Т. 198. С. 125-152.

[7] Schwartz L. Théorie des distributions. V. 2. Paris: Hermann, 1951.

[8] Стейн И., Вейс Г. Введение в гармонический анализ на евклидовых пространствах. М.: Мир, 1974.

[9] Соболев С. Л. Введение в теорию кубатурных формул. М.: Наука, 1974.

[10] Соболев С. Л . Некоторые применения функционального анализа в математической физике. М.: Наука, 1988.

[11] Duchon J. Interpolation des fonctions de deux variables suivant le principe de la flexion des plaques minces // RAIRO Modél. Math. Anal. Numér. 1976. V. 10. №12. P. 5-12.

[12] Duchon J. Sur l'erreur d'interpolation des fonctions de plusieurs variables par les $D^{m}$-splines // RAIRO Modél. Math. Anal. Numér. 1978. V. 12. №4. P. 325-334.

[13] Матвеев О.В. Аппроксимативные свойства интерполяционных $D^{m}$-сплайнов // Докл. AH CCCP. 1991. T. 321. № 1. C. 14-18.

[14] Madych W. R., Nelson S. A. Multivariate interpolation and conditionally positive definite functions // Approx. Theory Appl. (N. S.) 1988. V. 4. № 4. P. 77-89.

Институт математики и механики УрО РАН, г. Екатеринбург

Поступило

E-mail: matveev@apprekat.imm.intec.ru 